\title{
The Syntheses of Some Novel (Naphthalen-1-yl-selenyl)acetic Acid Derivatives
}

\author{
Serkan Yavuz ${ }^{1}$, Ali Dişli ${ }^{1}$, Yılmaz Yıldırır ${ }^{1, *}$ and Lemi Türker ${ }^{2}$ \\ ${ }^{1}$ Department of Chemistry, Faculty of Arts and Sciences, Gazi Universitesi, Teknik Okullar, 06500 \\ Ankara, Turkey \\ ${ }^{2}$ Department of Chemistry, Middle East Technical University, 06531 Ankara, Turkey \\ * Author to whom correspondence should be addressed; e-mail: yildirir@gazi.edu.tr
}

Received: 11 February 2005; in revised form: 29 April 2005 / Accepted: 30 April 2005 / Published: 31 August 2005

\begin{abstract}
Some new (naphthalen-1-yl-selenyl)acetic acids derivatives 7a-d have been synthesized by two different methods, using naphthylselenols or naphthylselenocyanates. The structures of the products were investigated by spectroscopic methods.
\end{abstract}

Keywords: Selenol, selenocyanate, naphthalene, selenium, (naphthalen-1-yl-selenyl)acetic acid.

\section{Introduction}

Selenium is an element which resembles sulfur in terms of its chemical properties. It has been successfully introduced into organic compounds either as an electrophile or as a nucleophile. Humans and animals need selenium for various biological functions which involve some organoselenium compounds [1]. It is also know that many selenium containing organic molecules are antibacterial and antifungal agents [2]. Organoselenium compounds are commonly employed as useful and powerful reagents in organic synthesis and many different organoselenium compounds have been synthesized $[3,4]$. Arylselenyl acetic acid derivatives are of interest due to their utility in some syntheses [5]. There are different approaches to the synthesis of arylselenyl acetic acid derivatives [6,7]. Two of these approaches are based on: i) arylselenols and ii) arylselenocyanates. The arylselenols are synthesized using Grignard reagents [8], whereas the arylselenocyanates are obtained from the reaction of aryldiazonium salts with potassium selenocyanate $[9,10]$. In this paper the naphtyl moiety has been introduced as the aryl group in the abovementioned compounds. 


\section{Results and Discussion}

In the present study, compounds 7a-d were synthesized starting from the corresponding naphtylselenol 3 or naphthylselenocyanates 6a-c. Schemes 1 and 2 outline the syntheses of these intermediates in the syntheses of the selenoacetic acids.

\section{Scheme 1}<smiles>Cc1ccc(Br)c2ccccc12</smiles>

1<smiles>Cc1ccc(SCC(=O)O)c2cc(Cl)ccc12</smiles>

3

In the second method (Scheme 2) the yields are generally low unless the medium is buffered ( $\mathrm{pH}=$ 5.5) by the addition of NaOAc. Most probably due to the hyperacidity, potassium selenocyanate decomposes to release $\mathrm{HCN}$.

\section{Scheme 2}

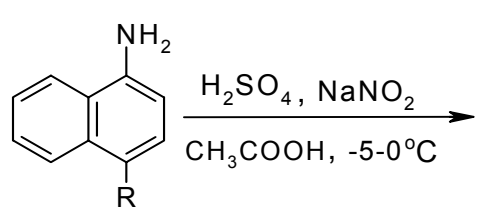

4a-c<smiles>[R]c1ccc([N+](=O)O)c2ccccc12</smiles>

5a-c<smiles>[R]c1ccc([Se])c2ccccc12</smiles>

6a-c

$\mathrm{R}=\mathrm{a}: \mathrm{H}, \mathrm{b}: \mathrm{NO}_{2}, \mathrm{c}: \mathrm{Br}$

The target (naphthalen-1-yl-selenyl)acetic acid derivatives are then synthesized either from the naphthalylselenol or naphthylselenocyanates with neutralized chloroacetic acid (Scheme 3).

Scheme 3

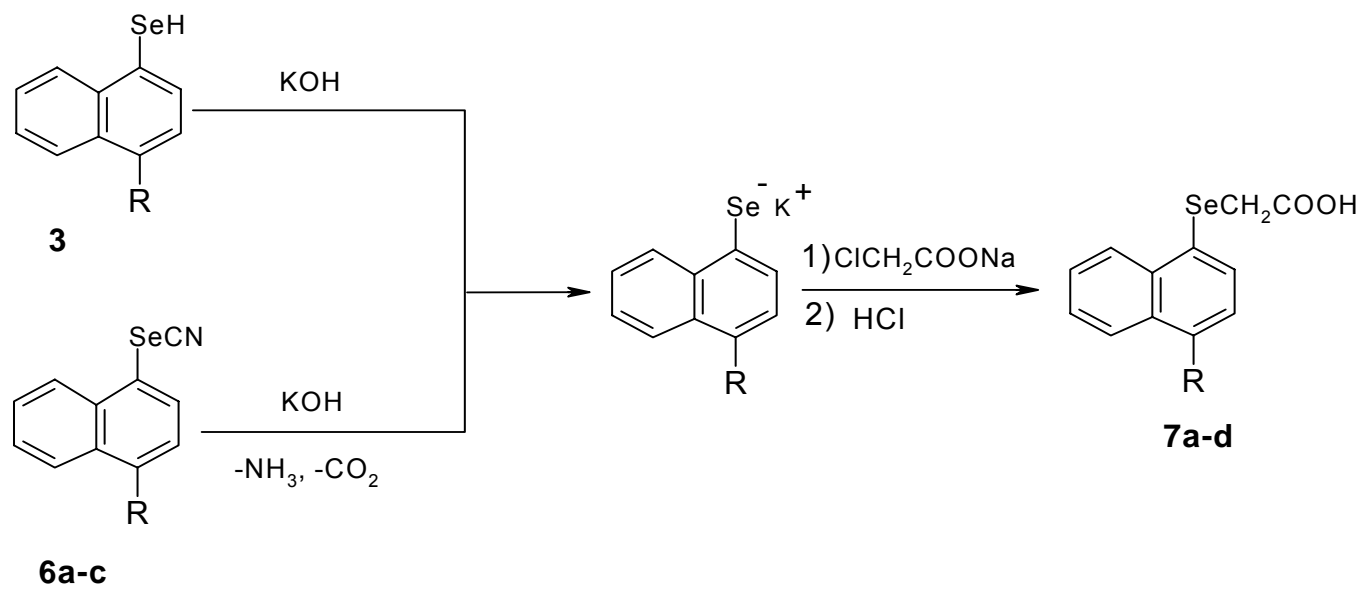

$\mathrm{R}=\mathrm{a}: \mathrm{H}, \mathrm{b}: \mathrm{NO}_{2}, \mathrm{c}: \mathrm{Br}, \mathrm{d}: \mathrm{CH}_{3}$ 
(Naphthalen-1-yl-selenyl)acetic acid (7a) was obtained both by Grignard synthesis [7] or via the corresponding diazonium salt. In each case the same product was obtained in comparable yields.

\section{Conclusions}

The intermediate products obtained in these syntheses are novel. They were isolated and their molecular structures identified. The final compounds 7a-d have some further synthetic potential in the direction of some heterocyclic compounds, which could be obtained by means of ring closure reactions such as Friedel Crafts reactions.

\section{Acknowledgements}

We are grateful to the Gazi University Research Foundation for financial support of this work (FEF 05/2002-19). In addition, we would like to thanks to the DPT Project (No: 2001K120590) for NMR and MS spectra measurements.

\section{Experimental}

\section{General}

Substituted naphthylamines, bromonaphthalenes and other chemicals were obtained from Merck. All mps were determined in sealed capillaries and are uncorrected. FT-IR spectra were recorded on a Mattson 1000 spectrometer as $\mathrm{KBr}$ pellets. ${ }^{1} \mathrm{H}-\mathrm{NMR}$ spectra were recorded on a Varian Gemini 300 (300 MHz) NMR spectrometer in $\mathrm{CDCl}_{3}$ and $\mathrm{CCl}_{4}$. Mass spectra measurements were recorded on a Thermo Finnigan Trace DSQ.

\section{4-Methyl-1-naphthylselenol (3)}

The Grignard reagent of 1-bromo-4-methyl naphthalene (3.9 $\mathrm{mL} ; 0.025 \mathrm{~mole})$ was prepared under a nitrogen atmosphere using dry ether as solvent. Selenium (grey, $1.975 \mathrm{~g}, 0.025$ mole) was added and the mixture refluxed for 2 hours. Dilute $\mathrm{HCl}$ solution was then added into the mixture. The ether layer was separated and evaporated under vacuum to yield the title compound as a viscous liquid (4.365 g, 0.020 mole, yield: 79\%); IR $v_{\max } / \mathrm{cm}^{-1}: 2048(\mathrm{Se}-\mathrm{H}), 3075(\mathrm{Ar}-\mathrm{H}), 2973(\mathrm{C}-\mathrm{H}) ;{ }^{1} \mathrm{H}-\mathrm{NMR}\left(\mathrm{CCl}_{4}\right) \delta_{\mathrm{H}}$ : $1.8(\mathrm{~s}, 1 \mathrm{H}) ; 2.5(\mathrm{~s}, 3 \mathrm{H}) ; 7.1-8.2(\mathrm{~m}, 6 \mathrm{H})$; MS for $\mathrm{C}_{11} \mathrm{H}_{10} \mathrm{Se}\left(\mathrm{M}^{+}\right)$: 222.1

1-Naphthylselenocyanate (6a). $\mathrm{NaNO}_{2}\left(0.17 \mathrm{~g} ; 0.0025\right.$ mole) was dissolved in cooled $\mathrm{H}_{2} \mathrm{SO}_{4}$. 1-Naphthylamine $\left(0.357 \mathrm{~g} ; 0.0025\right.$ mole) was dissolved in glacial acetic acid and cooled below $20{ }^{\circ} \mathrm{C}$. The solution of $\mathrm{H}_{2} \mathrm{SO}_{4}-\mathrm{NaNO}_{2}$ was dropwise added into the solution of $\alpha$-naphthylamine. The $\mathrm{pH}$ of the medium was adjusted to 5.5 by the addition of sodium acetate solution. Then $\mathrm{KSeCN}(0.36 \mathrm{~g}$; 0.0025 mole) was added portionwise. A brown precipitate formed, which was filtered by suction, dried and purified by crystallization. Yield: 44\%; m.p. 66-67 ${ }^{\circ} \mathrm{C}$ (ethanol) (lit. [11]: m.p. 69-70 ${ }^{\circ} \mathrm{C}$ ); IR $v_{\max } / \mathrm{cm}^{-1} 2158(\mathrm{SeC} \equiv \mathrm{N}), 3055(\mathrm{Ar}-\mathrm{H}) ;{ }^{1} \mathrm{H}-\mathrm{NMR}\left(\mathrm{CCl}_{4}\right)$ 7.3-8.5 (m, Ar-H); MS for $\mathrm{C}_{11} \mathrm{H}_{7} \mathrm{NSe}\left(\mathrm{M}^{+}\right)$: 232.9 
4-Nitro-1-naphthylselenocyanate (6b). Yield: 46\%; m.p. 88-89 ${ }^{\circ} \mathrm{C}$ (ethanol); IR $v_{\max } / \mathrm{cm}^{-1}: 2156$ $(\mathrm{SeC} \equiv \mathrm{N}), 3083(\mathrm{Ar}-\mathrm{H}) ;{ }^{1} \mathrm{H}-\mathrm{NMR}\left(\mathrm{CCl}_{4}\right) \delta_{\mathrm{H}}: 7.1-8.6(\mathrm{~m}, \mathrm{Ar}-\mathrm{H})$; MS for $\mathrm{C}_{11} \mathrm{H}_{6} \mathrm{~N}_{2} \mathrm{O}_{2} \mathrm{Se}\left(\mathrm{MH}^{+}\right): 279.0$

4-Bromo-1-naphthylselenocyanate (6c). Yield: 38\%; m.p.78-80 ${ }^{\circ} \mathrm{C}$ (ethanol); IR $v_{\max } / \mathrm{cm}^{-1}: 2152$ $(\mathrm{SeC} \equiv \mathrm{N}), 3075(\mathrm{Ar}-\mathrm{H}) ;{ }^{1} \mathrm{H}-\mathrm{NMR}\left(\mathrm{CCl}_{4}\right) \delta_{\mathrm{H}}: 7.2-8.4(\mathrm{~m}, \mathrm{Ar}-\mathrm{H})$

(Naphthalen-1-yl-selenyl)acetic acid (7a). $\mathrm{KOH}(3 \mathrm{M}, 30 \mathrm{~mL})$ was added to $6 \mathbf{a}$. This mixture was stirred under reflux for 2 hours in a water bath. Neutralized chloroacetic acid was added to the cooled mixture. The final mixture was boiled for 30 minutes. Then $\mathrm{HCl}$ was added to the cooled mixture until the precipitation was complete. The precipitate obtained was dried and purified by crystallization. Yield: 28\%; m.p. 65-66 ${ }^{\circ} \mathrm{C}\left(\mathrm{CCl}_{4}\right)$ (lit. [7]: m.p. 66-67 ${ }^{\circ} \mathrm{C}$; IR $v_{\max } / \mathrm{cm}^{-1}: 1703(\mathrm{C}=\mathrm{O}), 2816(\mathrm{C}-\mathrm{H}), 3040$ $(\mathrm{Ar}-\mathrm{H}), 3140(\mathrm{O}-\mathrm{H}) ;{ }^{1} \mathrm{H}-\mathrm{NMR}\left(\mathrm{CDCl}_{3}\right) \delta_{\mathrm{H}}: 3.5(\mathrm{~s}, 2 \mathrm{H}) ; 7.2-8.5(\mathrm{~m}, 7 \mathrm{H}) ; 9.6(\mathrm{~s}, 1 \mathrm{H})$; MS for $\mathrm{C}_{12} \mathrm{H}_{10} \mathrm{O}_{2} \mathrm{Se}\left(\mathrm{M}^{+}\right): 265.9$

The following compounds were similarly prepared:

(4-Nitronaphthalen-1-yl-selenyl)acetic acid (7b). Yield: 31\%; m.p. 91-93 ${ }^{\circ} \mathrm{C}\left(\mathrm{CCl}_{4}\right)$; IR $v_{\max } / \mathrm{cm}^{-1}$ : $1747(\mathrm{C}=\mathrm{O}), 2935(\mathrm{C}-\mathrm{H}), 3095(\mathrm{Ar}-\mathrm{H}), 3147(\mathrm{O}-\mathrm{H}) ;{ }^{1} \mathrm{H}-\mathrm{NMR}\left(\mathrm{CDCl}_{3}\right) \delta_{\mathrm{H}}: 3.7(\mathrm{~s}, 2 \mathrm{H}) ; 7.8-9.0(\mathrm{~m}$, $6 \mathrm{H}) ; 11.2(\mathrm{~s}, 1 \mathrm{H}) ; \mathrm{MS}$ for $\mathrm{C}_{11} \mathrm{H}_{6} \mathrm{~N}_{2} \mathrm{O}_{2} \mathrm{Se}\left(\mathrm{C}_{10} \mathrm{H}_{6} \mathrm{NO}_{2}{ }^{+}\right): 173.0$

(4-Bromonaphthalen-1-yl-selenyl)acetic acid (7c). Yield: 25\%; m.p. 94-95 ${ }^{\circ} \mathrm{C}$ (formic acid); IR $v_{\max } / \mathrm{cm}^{-1}: 1708(\mathrm{C}=\mathrm{O}), 2921(\mathrm{C}-\mathrm{H}), 3075(\mathrm{Ar}-\mathrm{H}), 3317(\mathrm{O}-\mathrm{H}) ;{ }^{1} \mathrm{H}-\mathrm{NMR}\left(\mathrm{CDCl}_{3}\right) \delta_{\mathrm{H}}: 3.2(\mathrm{~s}, 2 \mathrm{H})$; 7.6$8.8(\mathrm{~m}, 6 \mathrm{H}) ; 10.8(\mathrm{~s}, 1 \mathrm{H})$

(4-Methylnaphthalen-1-yl-selenyl)acetic acid (7d). $\mathrm{KOH}(3 \mathrm{M}, 30 \mathrm{~mL})$ was added to 3 . This mixture was stirred under reflux for 2 hours in a water bath. Neutralized chloroacetic acid was added into the cooled mixture. The final mixture was boiled for 30 minutes, and then $\mathrm{HCl}$ was added to the cooled mixture until the precipitation was complete. The precipitate obtained was dried and purified by crystallization. Yield: 38\%; m.p.72-73 ${ }^{\circ} \mathrm{C}\left(\mathrm{CCl}_{4}\right)$; IR $v_{\max } / \mathrm{cm}^{-1}: 1708(\mathrm{C}=\mathrm{O}), 2937(\mathrm{C}-\mathrm{H}), 3000(\mathrm{Ar}-\mathrm{H})$, $3120(\mathrm{O}-\mathrm{H})$; ${ }^{1} \mathrm{H}-\mathrm{NMR}\left(\mathrm{CDCl}_{3}\right) \delta_{\mathrm{H}}: 2.7(\mathrm{~s}, 3 \mathrm{H}), 3.5(\mathrm{~s}, 2 \mathrm{H}) ; 7.3-8.5(\mathrm{~m}, 6 \mathrm{H}) ; 10.1(\mathrm{~s}, 1 \mathrm{H})$; MS for $\mathrm{C}_{13} \mathrm{H}_{12} \mathrm{O}_{2} \mathrm{Se}\left(\mathrm{M}^{+}\right): 279.9$

\section{References}

1. Rayman, M.P. Brit. Med. J. 2000, 356, 233.

2. Günther, W.H.H. in Organic Selenium Compounds Their Chemistry and Biology; Klayman, D.L.; Günther, W.H.H. (Eds.); Wiley: New York, 1973; p. 30.

3. Xie, Y.; Short, M.D.; Cassidy P.B.; Roberts J.C. Bioorg. Med. Chem. Lett. 2001, 11, 2911.

4. Overvad K.; Bibl. Nutr. Diet. 1998, 54, 141.

5. Bellinger, N.; Cagniant, P. C.R. Acad. Sc. Paris 1969, 268, 1385.

6. Morgan, G.T.; Porrit, W.H. J. Chem. Soc. 1925, 1755.

7. Sjöberg, B.; Herdevall, S. Acta Chem. Scand. 1958, 12, 1347. 
8. Taboury, M.; Bull. Soc. Chim. France 1903, 29, 762.

9. Behagel, O.; Rollmann, M. J. Prakt. Chem. 1929, 123, 336.

10. Özkan, H.; Dişli, A.; Yıldırır, Y. Org. Prep. Proc. Int. 2004, 36, 161.

11. Murata, S.; Suzuki, C.; Inoue H.; Andoha Y.; Hayashi Y. Heterocycles, 1999, 52, 621.

Sample availability: Samples may be obtained from corresponding author.

(C) 2005 by MDPI (http://www.mdpi.org). Reproduction is permitted for noncommercial purposes. 